uncertain. Nonetheless, the present results justify further development of this exciting new technology. It is time for engineers and neurophysiologists to push the boundaries of how slow they can go.

Jed A. Hartings

College of Medicine of the University of Cincinnati,
Cincinnati, $\mathrm{OH}$, USA.

e-mail: jed.hartings@uc.edu

Published online: 31 December 2018

https://doi.org/10.1038/s41563-018-0272-5

References

1. Masvidal-Codina, E. et al. Nat. Mater. https://doi.org/10.1038/ s41563-018-0249-4 (2019).
2. Hébert, C. et al. Adv. Funct. Mater. 28, 1703976 (2018).

3. Kostarelos, K., Vincent, M., Hebert, C. \& Garrido, J. A Adv. Mater. 29, 1700909 (2017).

4. Dreier, J. P. Nat. Med. 17, 439-447 (2011).

5. Leao, A. A. J. Neurophysiol. 10, 409-414 (1947).

6. Dreier, J. P. et al. J. Cereb. Blood Flow Metab. 37, 1595-1625 (2017).

7. Lückl, J. et al. Brain 141, 1734-1752 (2018).

8. Dreier, J. P. et al. Ann. Neurol. 83, 295-310 (2018).

9. Hartings, J. A. et al. J. Cereb. Blood Flow Metab. 37, 1857-1870 (2017). 10. Li, C. et al. J. Neural. Eng. 13, 016008 (2016).

\title{
Taking the temperature of a sand pile
}

Tan he intuition, originated by Sam Edwards ${ }^{1}$, that granular materials can be described using the methods of statistical mechanics is an old one. It has a lot to recommend it. Like the fundamental states of matter, powders and other grainy systems are made up of interacting particles that have a vast array of configurations available to them, and which might therefore be best described by considering averages over ensembles.

But making the analogy concrete has proved difficult. States of matter may be described by equations of state that relate macroscopic thermodynamic variables such as pressure, volume and temperature, as in the well-known expression for an ideal gas. It is clear what volume (or density) might mean for granular materials, and they are subject too to a global confining pressure. But what is the temperature?

In the conventional sense, temperature is irrelevant to these systems. The grains are too large to undergo thermal fluctuations, so that in effect they are athermal: at zero temperature. A given configuration is immobile. What is more, there is generally a history-dependence and contingency to granular configurations, which may depend on exactly how they are prepared and loaded. So it is far from obvious whether a variable equivalent to temperature exists, or if so, what it is.

In the classical statistical mechanics of Boltzmann, temperature is defined as the inverse of the (partial) derivative of the system entropy with respect to energy, the latter being a conserved quantity. But in granular media, (internal) energy is not conserved because it is dissipated by friction as grains move. Edwards proposed that a temperature-like quantity might nonetheless be defined as the derivative of entropy with respect to volume, which he called the compactivity. At zero compactivity the system of grains will be at its densest random close packing.

This notion was soon refined to take into account the existence of the local force balance on each particle in a static, jammed state, leading to the definition of a tensor that represents a sum over these forces for all pairs of grains. A quantity that Edwards called the angoricity - the derivative of entropy with respect to this forcemoment tensor - then seemed a better candidate for the analogue of temperature ${ }^{2}$.

But is it? Bililign et al. ${ }^{3}$ have re-examined the issue experimentally, using as their model granular medium a system of around 900 hard circular photoelastic discs of two sizes (radii 5.5 and $7.7 \mathrm{~mm}$ ) confined between two plates and subjected to compressive or shear forces at the periphery. A flow of air eliminates friction with the confining plates - the discs float like table-hockey pucks. The stresses in the discs can be monitored using polarized light to reveal their photoelastic response, showing the networks of stress chains threading through this two-dimensional medium.

The results show that angoricity is after all not a valid state variable, being dependent on the loading protocol - whether it is, for example, uniaxial, biaxial or shear stress. But an alternative variable exists that doesn't have this dependence, namely the so-called keramicity. This is a quasigeometric factor related to the diagram of forces. This web can be represented as a tessellation of tiles, where each tile represents a particle and its edges have lengths proportional to the forces

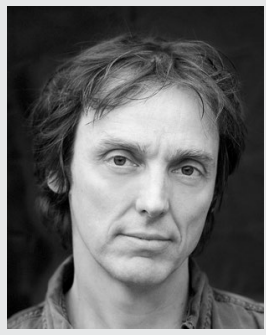

Philip Ball

acting on it $^{4}$. This is called a MaxwellCremona diagram.

The area of the tiling is a conserved quantity, and Bililign et al. show that the derivative of entropy with respect to this area, the keramicity (after the Greek word for tile), acts as a protocol-independent state variable analogous to temperature.

It sounds like a highly abstract quantity, but perhaps might become less so when we consider that, just as microscopic change in a gas is driven by thermal fluctuations, so microscopic rearrangements in a granular medium come from the network of forces that hold the grains in place. The system's freedom to explore configurational space is thus governed by geometry - of which the Maxwell-Cremona tessellation of stresses turns out to be the best characterization.

Published online: 19 February 2019 https://doi.org/10.1038/s41563-019-0306-7

References

1. Edwards, S. F. \& Oakeshott, R. B. S. Physica A 157 1080-1090 (1989).

2. Bi, D., Henkes, S., Daniels, K. E. \& Chakraborty, B. Annu. Rev. Condens. Matter Phys. 6, 63-83 (2015).

3. Bililign, E. S., Kollmer, J. E. \& Daniels, K. E. Phys. Rev. Lett. 122, 038001 (2019).

4. Tighe, B. P. \& Vlugt, T. J. H. J. Stat. Mech. 2011, P04002 (2011). 